\title{
Effective Elastoplastic Damage Mechanics for Fiber-reinforced Composites with Evolutionary Complete Fiber Debonding
}

\author{
J. W. JU* AND Y. F. Ko \\ Department of Civil and Environmental Engineering \\ University of California, Los Angeles, CA 90095-1593, USA \\ H. N. RUAN \\ Department of Civil and Environmental Engineering \\ Hohai University, Nanjing, P.R. China
}

\begin{abstract}
A micromechanical damage mechanics framework is proposed to predict the overall elastoplastic behavior and interfacial damage evolution of fiberreinforced ductile composites. Progressively debonded fibers are replaced by equivalent voids. The effective elastic moduli of three-phase composites, composed of a ductile matrix, randomly located yet unidirectionally aligned circular fibers, and voids, are derived by using a rigorous micromechanical formulation. In order to characterize the homogenized elastoplastic behavior, an effective yield criterion is derived based on the ensemble area averaging process and the first-order effects of eigenstrains. The resulting effective yield criterion, together with the overall associative plastic flow rule and the hardening law, constitutes the analytical framework for the estimation of effective elastoplastic damage responses of ductile composites containing both perfectly bonded and completely debonded fibers. An evolutionary interfacial fiber debonding process, governed by the internal stresses of fibers and interfacial strength, is incorporated into the proposed framework. The Weibull's function is employed to describe the varying probability of fiber debonding. Further, comparison between predictions and available experimental data are presented to illustrate the potential of the proposed methodology.
\end{abstract}

KEY WORDS: fiber-reinforced composites, micromechanics, damage mechanics, plasticity, interfacial debonding, homogenization, random media, probabilistic distribution.

*Professor and author to whom correspondence should be addressed. E-mail: juj@ucla.edu

International Journal of DAmage Mechanics, Vol. 15-July 2006 


\section{INTRODUCTION}

$\mathbf{F}$ IBER-REINFORCED DUCTILE MATRIX composites (FRDMCs) have been increasingly adopted or considered in engineering design and manufacturing due to their appealing mechanical properties. Matrix materials of FRDMCs are typically made of ductile metals or alloys with inelastic deformation and energy dissipation capabilities, such as aluminum, steel or titanium alloys. By contrast, fibers dispersed in the matrix generally behave elastically, which may be composed of carbon, boron or glass fibers, for instance. Many analytical methods were developed to predict the overall elastic moduli of FRDMCs; see, e.g., Hashin and Shtrikman (1962), Hashin and Rosen (1964), Hill (1964a,b), Hashin (1972), Willis (1981), and Ju and Zhang (1998). Other researchers proposed approaches to evaluate the elastoplastic behavior of fiber (or inclusion) reinforced ductile composites, such as Zhao and Weng (1990), DeBotton and Ponte Castañeda (1993), Doghri and Friebel (2005), and so on. In addition, Ju and Chen (1994c), developed a micromechanical formulation to predict the effective elastoplastic behavior of two-phase particle-reinforced ductile matrix composites (PRDMCs) under arbitrary loading histories by considering the first-order stress perturbations of elastic particles on the ductile matrix. Ju and Tseng (1996, 1997) further improved this work by incorporating second-order stress perturbations due to pairwise particle interactions based on the work of Ju and Chen (1994a, b). Moreover, Ju and Sun (1999), Ju and Zhang (2001), Sun and Ju (2004), extended the above micromechanical framework to predict the elastoplastic behavior of particle or fiber reinforced ductile composites.

However, damage in FRDMCs may occur inevitably during the history of loading, thus affecting the behavior of composites. In the last decade, extensive literatures were published on damage in composites; see, e.g., $\mathrm{Ju}$ (1991a, b), Ju and Lee (1991), Lee and Ju (1991), Ju and Tseng (1992), Ju and Chen (1994d,e), Tohgo and Weng (1994), Ju and Tseng (1995), Zhao and Weng (1995), Ju (1996), Voyiadjis and Park (1996), and Lissenden (1996), etc. Experiments have illustrated several different damage mechanisms, such as damage in fibers or the matrix, and the interfacial debonding between the matrix and fibers (inclusions). A volume integral equation method was used to investigate the damage evolution in unidirectional $\mathrm{SiC} / \mathrm{Ti}$ composites under transverse loading by Lee and Mal (1998). The most likely mechanism of damage here is the initiation of partial fiber debonding followed by transverse cracking in brittle matrix composites or plastic yielding in ductile matrix composites. Furthermore, experimental evidence (Nimmer et al., 1991) showed that the micromechanism of damage is often associated with the formation and 


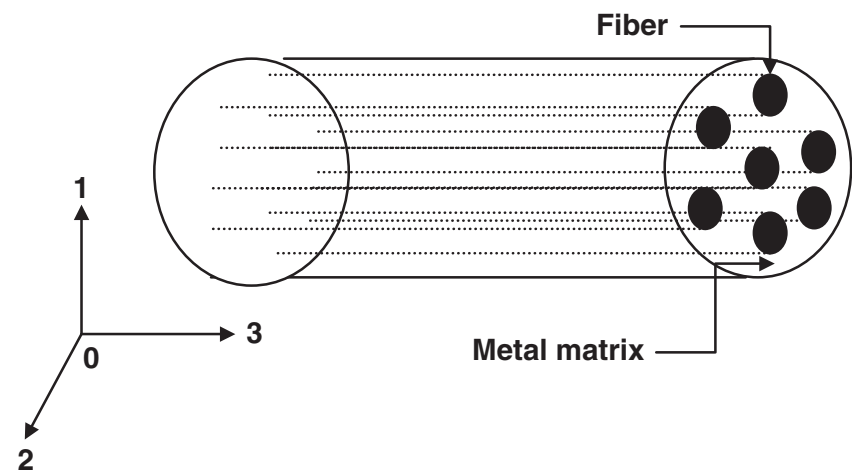

Figure 1. Schematic plot of a composite reinforced by unidirectionally aligned yet randomly located long circular fibers.

growth of voids at the matrix-fiber interfaces under transverse loading. Therefore, interfacial debonding is the dominant damage mechanism in this study.

The main objective of the present work is to develop an elastoplastic damage mechanics framework for fiber-reinforced ductile composites under transverse biaxial loading. The fibers are assumed to be elastic circular cylinders, which are randomly located in the matrix and unidirectionally aligned; see Figure 1. The ductile (metal) matrix behaves elastoplastically under arbitrary loading histories. All fibers are initially embedded firmly in the matrix. Once the composites are loaded to a certain level, interfacial debonding may occur around fiber interfaces. For simplicity, debonded fibers are regarded as cylindrical voids; see Figure 2. The overall elastic material properties of the three-phase composites are statistically homogeneous and transversely isotropic.

\section{EFFECTIVE ELASTIC MODULI OF 3-PHASE FIBER COMPOSITES}

Let us consider an initially perfectly bonded two-phase composite consisting of an elastic matrix (phase 0) with the bulk modulus $k_{0}$ and shear modulus $\mu_{0}$, and randomly located yet unidirectionally aligned elastic circular fibers (phase 1) with the bulk modulus $k_{1}$, and shear modulus $\mu_{1}$. When loadings or deformations are applied and gradually increased, some fibers may progressively experience interfacial debonding. For simplicity, these debonded fibers are regarded as cylindrical voids (phase 2 ) in the current framework. For FRDMCs, the plane-strain condition can be assumed; i.e., a generalized two-dimensional plane-strain problem. 


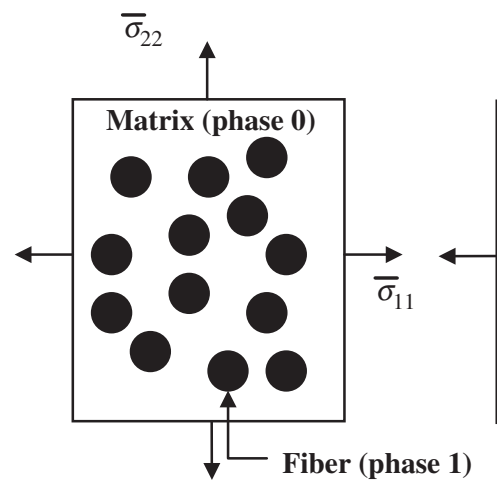

(a)

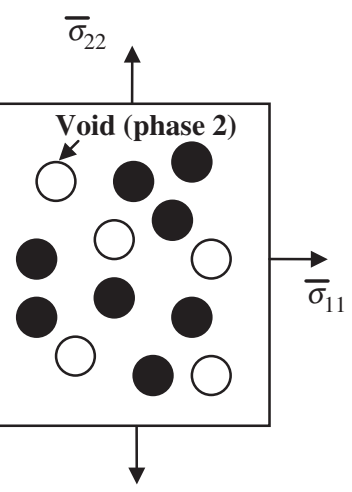

(b)<smiles>[123I]C[Tl]</smiles>

Figure 2. A schematic diagram of FRDMCs subjected to biaxial transverse tensile normal loading: (a) the initial state (undamaged) and (b) the equivalent damaged state (voids).

The relationship between the stress tensor $\boldsymbol{\sigma}$ and the strain tensor $\boldsymbol{\varepsilon}$ at any point $\mathbf{x}$ in the $q$-phase $(q=0,1,2)$ is governed by

$$
\boldsymbol{\sigma}(\mathbf{x})=\mathbf{C}_{q}: \varepsilon(\mathbf{x})
$$

where ':' denotes the tensor contraction and $\mathbf{C}_{q}$ is the elasticity tensor of the $q$-phase. At the macroscopic level, the overall effective elasticity tensor $\mathbf{C}_{*}$ is defined as the relation between the average (homogenized) stress and strain:

$$
\overline{\boldsymbol{\sigma}}=\mathbf{C}_{*}: \overline{\boldsymbol{\varepsilon}}
$$

In order to derive the in-plane and out-of-plane effective moduli of fiberreinforced composites, three-dimensional analysis is conducted. Effective elastic moduli of multiphase composites containing randomly located, unidirectionally aligned elastic ellipsoids were explicitly derived by Ju and Chen (1994a,b) accounting for far-field perturbations and near-field pairwise particle interactions. For multi-phase composites, the effective (semi-interacting) elasticity tensor $\mathbf{C}_{*}$ reads (Ju and Chen, 1994a)

$$
\mathbf{C}_{*}=\mathbf{C}_{0} \bullet\left\{\mathbf{I}+\mathbf{B} \bullet(\mathbf{I}-\mathbf{S} \bullet \mathbf{B})^{-1}\right\}
$$


where $\mathbf{C}_{0}$ is the matrix elasticity tensor, $\mathbf{I}$ is the fourth-order identity tensor, '•' denotes the tensor multiplication, and $\mathbf{B}$ takes the form

$$
\mathbf{B}=\sum_{q=1}^{n} \phi_{q}\left(\mathbf{S}+\mathbf{A}_{q}\right)^{-1}
$$

Here, $n$ signifies the number of inclusion phases of different material properties, and $\phi_{q}$ denotes the volume fraction of the $q$-phase. The fourthrank tensor $\mathbf{A}_{q}$ is defined as

$$
\mathbf{A}_{q} \equiv\left[\mathbf{C}_{q}-\mathbf{C}_{0}\right]^{-1} \bullet \mathbf{C}_{0}
$$

Since the phase 2 contains voids, we have $\mathbf{A}_{2}=-\mathbf{I}$. The components of Eshelby's tensor $\mathbf{S}$ for an ellipsoidal inclusion are given by Mura (1987); $\mathrm{Ju}$ and Sun (1999, 2001), and Sun and Ju (2001). Following the method proposed by Ju and Sun (2001), with the axes 1 and 2 of the ellipsoid being equal and the 3rd axis going to infinity, we obtain the fourth-rank Eshelby's tensor for a cylindrical inclusion as follows

$$
S_{i j k l}=\frac{1}{4\left(1-v_{0}\right)}\left\{S_{\mathrm{IK}}^{(1)} \delta_{i j} \delta_{k l}+S_{\mathrm{IJ}}^{(2)}\left(\delta_{i k} \delta_{j l}+\delta_{i l} \delta_{j k}\right)\right\}
$$

where $v_{0}$ denotes Poisson's ratio of the matrix, and $\delta_{i j}$ signifies the Kronecker delta. The components of the second-rank tensors $S_{\mathrm{IK}}^{(1)}$ and $S_{\mathrm{IJ}}^{(2)}$ are given by

$$
\begin{aligned}
& S_{11}^{(1)}=S_{22}^{(1)}=S_{12}^{(1)}=S_{21}^{(1)}=\frac{1}{2}\left(4 v_{0}-1\right), \quad S_{13}^{(1)}=S_{23}^{(1)}=2 v_{0}, \\
& S_{31}^{(1)}=S_{32}^{(1)}=S_{33}^{(1)}=0, \quad S_{11}^{(2)}=S_{22}^{(2)}=S_{12}^{(2)}=S_{21}^{(2)}=\frac{1}{2}\left(3-4 v_{0}\right), \\
& S_{13}^{(2)}=S_{23}^{(2)}=S_{31}^{(2)}=S_{32}^{(2)}=\left(1-v_{0}\right), \quad S_{33}^{(2)}=0
\end{aligned}
$$

Substituting Equation (7) into Equation (6) yields the same Eshelby's tensor for the cylindrical inclusion given by Mura (1987). Applying Equations (3)-(7), we can therefore derive the in-plane and out-of-plane effective elastic moduli of debonded fiber-reinforced composites. That is, the tensor $\mathbf{C}_{*}$ reads

$$
\mathbf{C}_{*}=\left\{C_{\mathrm{IK}}^{(1)} \delta_{i j} \delta_{k l}+C_{\mathrm{IJ}}^{(2)}\left(\delta_{i k} \delta_{j l}+\delta_{i l} \delta_{j k}\right)\right\}
$$


where

$$
\mathrm{C}_{\mathrm{IK}}^{(1)}=\sum_{n=1}^{3} \lambda_{0} W_{n \mathrm{~K}}^{(1)}+2 \mu_{0} W_{\mathrm{IK}}^{(1)}+2 \lambda_{0} W_{\mathrm{KK}}^{(2)}, \quad C_{\mathrm{IJ}}^{(2)}=2 \mu_{0} W_{\mathrm{IJ}}^{(2)}
$$

Here, $\lambda_{0}$ and $\mu_{0}$ are the Lame's constants of matrix, and

$$
\begin{aligned}
W_{\mathrm{IK}}^{(1)} & =\sum_{m=1}^{3} \sum_{r=1}^{2} \frac{\phi_{r} Y_{\mathrm{I} m}^{r}}{\Psi_{\mathrm{II}}^{r}} \frac{Z_{m \mathrm{~K}}}{2 Q_{m m}^{(2)}}-2 \sum_{r=1}^{2} \frac{\phi_{r}}{2 \Psi_{\mathrm{II}}^{r}} \frac{Z_{\mathrm{IK}}}{2 Q_{\mathrm{II}}^{(2)}}-2 \sum_{r=1}^{2} \frac{\phi_{r} Y_{\mathrm{IK}}^{r}}{\Psi_{\mathrm{II}}^{r}} \frac{1}{4 Q_{\mathrm{KK}}^{(2)}} \\
W_{\mathrm{IJ}}^{(2)} & =2 \sum_{r=1}^{2} \frac{\phi_{r}}{2 \Psi_{\mathrm{II}}^{r}} \frac{1}{4 Q_{\mathrm{IJ}}^{(2)}}+\frac{1}{2}
\end{aligned}
$$

in which

$$
\begin{gathered}
\left\{\begin{array}{l}
Z_{\mathrm{I} 1} \\
Z_{\mathrm{I} 2} \\
Z_{\mathrm{I} 3}
\end{array}\right\}=\left[\begin{array}{ccc}
Q_{11}^{(1)}+2 Q_{11}^{(2)} & Q_{21}^{(1)} & Q_{31}^{(1)} \\
Q_{12}^{(1)} & Q_{22}^{(1)}+2 Q_{22}^{(2)} & Q_{32}^{(1)} \\
Q_{13}^{(1)} & Q_{23}^{(1)} & Q_{33}^{(1)}+2 Q_{33}^{(2)}
\end{array}\right]^{-1}\left\{\begin{array}{c}
Q_{\mathrm{I}}^{(1)} \\
Q_{\mathrm{I} 2}^{(1)} \\
Q_{\mathrm{I}}^{(1)}
\end{array}\right\} \\
Q_{\mathrm{IK}}^{(1)}=\frac{1}{4\left(1-v_{0}\right)}\left[\sum_{m=1}^{3} S_{\mathrm{Im}}^{(1)} \sum_{r=1}^{2} \frac{\phi_{r} Y_{m \mathrm{~K}}^{r}}{\Psi_{\mathrm{mm}}^{r}}+2 S_{\mathrm{II}}^{(2)} \sum_{r=1}^{2} \frac{\phi_{r} Y_{\mathrm{IK}}^{r}}{\Psi_{\mathrm{II}}^{r}}-2 S_{\mathrm{IK}}^{(2)} \sum_{r=1}^{2} \frac{\phi_{r}}{2 \Psi_{\mathrm{KK}}^{r}}\right]
\end{gathered}
$$


Following Hashin's (1972) framework, the five effective elastic moduli for three-phase composites can be derived. Accordingly, the two in-plane elastic constants (the effective transverse bulk and shear moduli) can be expressed as

$$
k_{T}^{*}=C_{11}^{(1)}+C_{11}^{(2)}, \quad \mu_{T}^{*}=C_{11}^{(2)}
$$

Moreover, the three out-of-plane effective elastic constants (the effective axial shear modulus, effective axial Young's modulus, and effective axial Poisson's ratio) read

$$
\mu_{A}^{*}=C_{13}^{(2)}, \quad E_{A}^{*}=C_{33}^{(1)}+2 C_{33}^{(2)}-\frac{\left(C_{13}^{(1)}\right)^{2}}{C_{11}^{(1)}+C_{11}^{(2)}}, \quad v_{A}^{*}=\frac{C_{13}^{(1)}}{2\left(C_{11}^{(1)}+C_{11}^{(2)}\right)}
$$

Alternatively, the in-plane overall elastic constants can also be represented by the effective transverse Young's modulus and effective Poisson's ratio:

$$
E_{T}^{*}=\frac{4 k_{T}^{*} \mu_{T}^{*}}{k_{T}^{*}+\Psi \mu_{T}^{*}}, \quad v_{T}^{*}=\frac{k_{T}^{*}-\Psi \mu_{T}^{*}}{k_{T}^{*}+\Psi \mu_{T}^{*}}, \quad \text { where } \Psi=1+\frac{4\left(v_{A}^{*}\right)^{2} k_{T}^{*}}{E_{A}^{*}}
$$

It is noteworthy that effective elastic moduli of three-phase composites derived in the present work fall within Hashin's (1972) variational bounds. Further, Hashin's (1972) variational lower bound of effective elastic moduli of two-phase composites can also be recovered.

\section{ELASTOPLASTIC DAMAGE BEHAVIOR OF FIBER COMPOSITES}

\section{Basic Analysis}

In this section, we consider the homogenized elastoplastic-damage responses of fiber-reinforced ductile composites with interfacial debonding. The composites initially feature perfect interfacial bonding between fibers and the matrix, but thereafter progressively debond into three-phase composites. That is, a two-phase composite may gradually become a three-phase composite consisting of the matrix, perfectly bonded fibers, and debonded fibers. In what follows, we will regard debonded fibers as cylindrical voids. For simplicity, the von Mises yield criterion with an isotropic hardening law is considered for the matrix. Extension of the present framework to more general yield criteria and hardening laws, 
nevertheless, is relatively straightforward. Accordingly, at any matrix material point, the stress $\sigma$ and the equivalent plastic strain $\bar{e}^{p}$ must satisfy the following yield function:

$$
F\left(\boldsymbol{\sigma}, \bar{e}^{p}\right)=H(\boldsymbol{\sigma})-K^{2}\left(\bar{e}^{p}\right) \leq 0
$$

in which $K\left(\bar{e}^{p}\right)$ is the isotropic hardening function of the matrix-only material. The stress tensor $\boldsymbol{\sigma}$ for the current generalized plane-strain problem reads

$$
\boldsymbol{\sigma}=\left(\begin{array}{ccc}
\sigma_{11} & \sigma_{12} & 0 \\
\sigma_{21} & \sigma_{22} & 0 \\
0 & 0 & \sigma_{33}
\end{array}\right), \quad \sigma_{33}=v_{A}^{*}\left(\sigma_{11}+\sigma_{22}\right)
$$

Furthermore, $H(\boldsymbol{\sigma}) \equiv \boldsymbol{\sigma}: \mathbf{I}_{\mathrm{d}}: \boldsymbol{\sigma}$ denotes the square of the deviatoric stress norm, where $\mathbf{I}_{\mathrm{d}}$ signifies the deviatoric part of the fourth-rank identity tensor I; i.e.,

$$
\mathbf{I}_{\mathrm{d}} \equiv \mathbf{I}-\frac{1}{3} \mathbf{1} \otimes \mathbf{1}
$$

in which 1 represents the second-rank identity tensor, and ' $\otimes$ ' denotes the tensor expansion. The total strain $\boldsymbol{\varepsilon}$ can be decomposed into two parts: $\varepsilon=\varepsilon^{e}+\varepsilon^{p}$, where $\varepsilon^{e}$ is the elastic strain of the matrix and fibers, and $\varepsilon^{p}$ represents the stress-free plastic strain in the matrix only. In order to solve the elastoplastic response exactly, the stress at any local point has to be solved and then used to determine the plastic response through the local yield criterion for all possible (probabilistic) configurations. This approach is generally infeasible due to the complexity of statistical and microstructural information. Instead, we employ a framework in which an ensembleaveraged yield criterion is constructed for composites. The methodology is parallel to the work of Ju and Chen (1994c) and Ju and Lee (2000, 2001), Sun et al. (2003a), Sun et al. (2003b), Liu et al. (2004), in which only the first-order effects are considered in the formulation of effective plastic response. However, by means of this method, we can achieve a feasible elastoplastic damage formulation for fiber composites.

\section{A First-order Formulation of the Stress Norm Accounting for Damage}

In this work, small strains are assumed and therefore the statistical microstructure of fibers embedded in a ductile matrix remains essentially 
the same. Therefore, the microstructure is taken as statistically homogeneous and isotropic with virtually constant volume fraction for the summation of fibers and voids during the deformation process. Furthermore, both fibers and voids are considered as cylinders of uniform size. The extension to accommodate different or distributed sizes of fibers can be derived with increased complexity.

Following $\mathrm{Ju}$ and Chen (1994c), Ju and Lee (2000, 2001), and Ju and Zhang (2001), we denote $H(\mathbf{x} \mid g)$ the square of the 'current stress norm' at the local point $\mathbf{x}$, which determines the plastic strain in a debonded FRDMC for a given phase configuration $g$. Since there is no plastic strain in the elastic fibers or voids, $H(\mathbf{x} \mid g)$ can be written as

$$
H(\mathbf{x} \mid g)=\left\{\begin{array}{cl}
\boldsymbol{\sigma}(\mathbf{x} \mid g): \mathbf{I}_{\mathrm{d}}: \boldsymbol{\sigma}(\mathbf{x} \mid g), & \text { if } \mathbf{x} \text { in the matrix } \\
0, & \text { otherwise }
\end{array}\right.
$$

In addition, $\langle H\rangle_{m}(\mathbf{x})$ is defined as the ensemble average of $H(\mathbf{x} \mid g)$ over all possible realization where $\mathbf{x}$ is in the matrix phase. Here, the angled bracket $\langle\cdot\rangle$ signifies the ensemble average operator. Let $P\left(g_{q}\right)$ be the probability density function for finding the $q$-phase $(q=1,2)$ configuration $g_{q}$ in the composites. $\langle H\rangle_{m}(\mathbf{x})$ can be obtained by integrating $H$ over all possible fiber and void configurations (for a point $\mathbf{x}$ in the matrix):

$$
\langle H\rangle_{m}=H^{\mathrm{o}}+\int_{g_{1}}\left\{H\left(\mathbf{x} \mid g_{1}\right)-H^{\mathrm{o}}\right\} P\left(g_{1}\right) \mathrm{d} g_{1}+\int_{g_{2}}\left\{H\left(\mathbf{x} \mid g_{2}\right)-H^{\circ}\right\} P\left(g_{2}\right) \mathrm{d} g_{2}
$$

where $H^{\circ}$ is the square of the far-field stress norm in the matrix: $H^{\mathrm{o}}=\boldsymbol{\sigma}^{\mathrm{o}}: \mathbf{I}_{\mathrm{d}}: \boldsymbol{\sigma}^{\mathrm{o}}$. The total stress at any point $\mathbf{x}$ in the matrix is the superposition of the far-field stress $\sigma^{\mathrm{o}} \equiv C_{0}: \boldsymbol{\varepsilon}^{\mathrm{o}}$ and the perturbed stress $\sigma^{\prime}$ due to the presence of the fibers and voids: $\sigma(\mathbf{x})=\sigma^{\circ}+\sigma^{\prime}(\mathbf{x})$, where $\boldsymbol{\sigma}^{\prime}$ is

$$
\boldsymbol{\sigma}^{\prime}(\mathbf{x}) \equiv \mathbf{C}_{0}: \int_{A} \mathbf{G}\left(\mathbf{x}-\mathbf{x}^{\prime}\right): \boldsymbol{\varepsilon}_{1}^{*}\left(\mathbf{x}^{\prime}\right) \mathrm{d} \mathbf{x}^{\prime}+\mathbf{C}_{0}: \int_{A} \mathbf{G}\left(\mathbf{x}-\mathbf{x}^{\prime}\right): \boldsymbol{\varepsilon}_{2}^{*}\left(\mathbf{x}^{\prime}\right) \mathrm{d} \mathbf{x}^{\prime}
$$

Here, $\boldsymbol{\varepsilon}^{\mathrm{o}}$ is the elastic strain field induced by the far-field loading, $\boldsymbol{\varepsilon}_{q}^{*}\left(\mathbf{x}^{\prime}\right)$ denotes the elastic eigenstrain in the $q$-phase $(q=1,2), \mathbf{x}^{\prime}$ resides in either a fiber or a void, and $A$ is the statistically representative area element (infinitely large compared with inhomogeneities, and without any prescribed displacement boundary conditions). Equation (26) represents the method 
of Green's function, with the indicial components of the fourth-rank tensor $G$ as

$$
G_{i j k l}\left(\mathbf{x}-\mathbf{x}^{\prime}\right)=\frac{1}{4 \pi\left(1-v_{0}\right) r^{2}} F_{i j k l}\left(-8,2 v_{0}, 2,2-4 v_{0},-1+2 v_{0}, 1-2 v_{0}\right)
$$

where $\mathbf{r} \equiv \mathbf{x}-\mathbf{x}^{\prime}, \mathbf{r} \equiv\|\mathbf{r}\|$, and the indices $i, j, k$, and $l$ are from 1 to 2 . The components of the fourth-rank tensor $\mathbf{F}$ - which depends on six scalar quantities $B_{1}, B_{2}, B_{3}, B_{4}, B_{5}, B_{6}-$ are defined by

$$
\begin{aligned}
F_{i j k l}\left(B_{\mathrm{m}}\right) \equiv & B_{1} n_{i} n_{j} n_{k} n_{l}+B_{2}\left(\delta_{i k} n_{j} n_{l}+\delta_{i l} n_{j} n_{k}+\delta_{j k} n_{i} n_{l}+\delta_{j l} n_{i} n_{k}\right) \\
& +B_{3} \delta_{i j} n_{k} n_{l}+B_{4} \delta_{k l} n_{i} n_{j}+B_{5} \delta_{i j} \delta_{k l}+B_{6}\left(\delta_{i k} \delta_{j l}+\delta_{i l} \delta_{j k}\right)
\end{aligned}
$$

with the unit normal vector $\mathbf{n} \equiv \mathbf{r} / \mathrm{r}$ and the index $\mathrm{m}=1$ to 6 . The unknown elastic eigenstrain $\boldsymbol{\varepsilon}_{q}^{*}(\mathbf{x})$ within the $q$-phase can be solved by the integral equation obtained from the celebrated Eshelby's equivalence principle (Eshelby, 1957). The outcome is

$$
-\mathbf{A}_{q}: \boldsymbol{\varepsilon}_{q}^{*}(\mathbf{x})=\boldsymbol{\varepsilon}^{\mathrm{o}}+\int_{A} \mathbf{G}\left(\mathbf{x}-\mathbf{x}^{\prime}\right): \boldsymbol{\varepsilon}_{q}^{*}\left(\mathbf{x}^{\prime}\right) \mathrm{d} x^{\prime}
$$

According to Eshelby (1957), the eigenstrain for a single ellipsoidal inclusion is uniform for the interior points of an isolated inclusion. Consequently, the constant eigenstrain can be moved out of the integral in Equation (26) in accordance with the first-order approximation approach proposed by $\mathrm{Ju}$ and Chen (1994c). It is noted that, for the first-order approximation method, the local interaction among fibers and/or voids are neglected in the process of collecting the perturbations of the stresses at a local matrix point. Therefore, the perturbed stresses for any matrix point $\mathbf{x}$ due to an isolated $q$-phase inhomogeneity centered at $\mathbf{x}_{q}^{(1)}$ takes the form

$$
\boldsymbol{\sigma}^{\prime}\left(\mathbf{x} \mid \mathbf{x}_{q}^{(1)}\right)=\left[\mathbf{C}_{0} \cdot \overline{\mathbf{G}}\left(\mathbf{x}-\mathbf{x}_{q}^{(1)}\right)\right]: \varepsilon_{q}^{* \circ}
$$

where $\boldsymbol{\varepsilon}_{q}^{* \mathrm{O}}$ is the solution of the eigenstrain $\boldsymbol{\varepsilon}_{q}^{*}$ for the single inclusion problem of the $q$-phase, and

$$
\overline{\mathbf{G}}\left(\mathbf{x}-\mathbf{x}_{q}^{(1)}\right) \equiv \int_{\Omega_{q}^{(1)}} \mathbf{G}\left(\mathbf{x}-\mathbf{x}^{\prime}\right) \mathrm{d} \mathbf{x}^{\prime}
$$


for $\mathbf{x} \notin \Omega_{q}^{(1)}$ in which $\Omega_{q}^{(1)}$ is the single inhomogeneity domain centered at $\mathbf{x}_{q}^{(1)}$ in the $q$-phase. Alternatively, we can write

$$
\overline{\mathbf{G}}\left(\mathbf{r}_{q}\right)=\frac{1}{8\left(1-v_{0}\right)}\left(\rho_{q}^{2} \mathbf{H}^{1}+\frac{\rho_{q}^{4}}{2} \mathbf{H}^{2}\right)
$$

The components of $\mathbf{H}^{1}$ and $\mathbf{H}^{2}$ are given by

$$
\begin{aligned}
& \mathbf{H}_{i j k l}^{1}\left(\mathbf{r}_{q}\right) \equiv 2 F_{i j k l}\left(-8,2 v_{0}, 2,2-4 v_{0},-1+2 v_{0}, 1-2 v_{0}\right) \\
& \mathbf{H}_{i j k l}^{2}\left(\mathbf{r}_{q}\right) \equiv 2 F_{i j k l}(24,-4,-4,-4,1,1)
\end{aligned}
$$

where $\mathbf{r}_{q}=\mathbf{x}-\mathbf{x}_{q}^{(1)}, \mathbf{r}_{q} \equiv\left\|\mathbf{r}_{q}\right\|, \rho_{q}=a / r_{q}$, and $a$ is the radius of a circular fiber or void.

Moreover, the 'first-order' eigenstrain $\varepsilon_{q}^{* 0}$ in Equation (30) is given by (see, e.g., Ju and Chen, 1994c)

$$
\boldsymbol{\varepsilon}_{q}^{* \mathrm{o}}=-\left(\mathbf{A}_{q}+\mathbf{S}\right)^{-1}: \boldsymbol{\varepsilon}^{\mathrm{o}}, \quad q=1,2
$$

where $\mathbf{S}$ is the Eshelby's tensor for a cylindrical inclusion. Under the planestrain condition, the components of $\mathbf{S}$ take the form (Ju and Zhang, 1998):

$$
S_{i j k l}=\frac{1}{8\left(1-v_{0}\right)}\left\{\left(4 \nu_{0}-1\right) \delta_{i j} \delta_{k l}+\left(3-4 v_{0}\right)\left(\delta_{i k} \delta_{j l}+\delta_{i l} \delta_{j k}\right)\right\}, \quad i, j, k, l=1,2
$$

\section{A First-order Formulation of the Effective Elastoplastic Damage Behavior of Composites}

As indicated before, a matrix point receives the perturbations from fibers and voids. Therefore, the ensemble-average stress norm for any matrix point $\mathbf{x}_{m}$ can be evaluated by collecting and summing up all the current stress norm perturbations produced by any typical fiber centered at $\mathbf{x}_{1}^{(1)}$ in the fiber domain and any typical void centered at $\mathbf{x}_{2}^{(1)}$ in the void domain, and averaging over all possible locations of $\mathbf{x}_{1}^{(1)}$ and $\mathbf{x}_{2}^{(1)}$. As a result, we arrive at

$$
\begin{aligned}
\langle H\rangle_{m}(\mathbf{x}) \cong & H^{\mathrm{o}}+\int_{\left|\mathbf{x}-\mathbf{x}_{1}^{(1)}\right|>a}\left\{H\left(\mathbf{x} \mid \mathbf{x}_{1}^{(1)}\right)-H^{\mathrm{o}}\right\} P\left(\mathbf{x}_{1}^{(1)}\right) \mathrm{d} \mathbf{x}_{1}^{(1)} \\
& +\int_{\left|\mathbf{x}-\mathbf{x}_{2}^{(1)}\right|>a}\left\{H\left(\mathbf{x} \mid \mathbf{x}_{2}^{(1)}\right)-H^{\mathrm{o}}\right\} P\left(\mathbf{x}_{2}^{(1)}\right) \mathrm{d} \mathbf{x}_{2}^{(1)}+\cdots
\end{aligned}
$$


where $P\left(\mathbf{x}_{1}^{(1)}\right)$ and $P\left(\mathbf{x}_{2}^{(1)}\right)$ denote the probability density functions for finding a fiber centered at $\mathbf{x}_{1}^{(1)}$ and a void centered at $\mathbf{x}_{2}^{(1)}$, respectively. In the absence of actual microstructural evidence, $P\left(\mathbf{x}_{1}^{(1)}\right)$ and $P\left(\mathbf{x}_{2}^{(1)}\right)$ are assumed to be statistically homogeneous, isotropic and uniform for simplicity. That is, we assume that the probability density functions are $P\left(\mathbf{x}_{1}^{(1)}\right)=\left(N_{1} / A\right)$ and $P\left(\mathbf{x}_{2}^{(1)}\right)=\left(N_{2} / A\right)$, where $N_{1}$ and $N_{2}$ are the total numbers of fibers and voids, respectively, dispersed in a representative area element $A$. If detailed spatial distribution of fibers can be provided, suitable probability distribution function can be readily implemented under the current framework. Unlike the effective medium methods (e.g., the self-consistent method, the generalized self-consistent method, the differential scheme, and the Mori-Tanaka method), where neither inclusion locations nor their relative configurations are taken into account, the present framework considers randomly located inclusions by employing some approximations. Further, owing to the assumed statistical isotropy and uniformity, Equation (37) can be recast as:

$$
\begin{aligned}
\langle H\rangle_{m}(\mathbf{x}) \cong & H^{\mathrm{o}}+\frac{N_{1}}{A} \int_{a}^{\infty} r_{1} \mathrm{~d} r_{1} \int_{0}^{2 \pi}\left\{H\left(\mathbf{r}_{1}\right)-H^{\circ}\right\} \mathrm{d} \theta \\
& +\frac{N_{2}}{A} \int_{a}^{\infty} r_{2} \mathrm{~d} r_{2} \int_{0}^{2 \pi}\left\{H\left(\mathbf{r}_{2}\right)-H^{\circ}\right\} \mathrm{d} \theta+\cdots
\end{aligned}
$$

where $\theta$ is the polar angle of circular fiber or void centered at $\mathbf{x}_{q}^{(1)}$.

Using Equations (34) and (35) in Ju and Zhang (1998) and the perturbed stresses given in Equation (30), we obtain the ensemble-averaged current stress norm at any matrix point:

$$
\langle H\rangle_{m}(\mathbf{x})=\boldsymbol{\sigma}^{\mathrm{o}}: \mathbf{T}: \boldsymbol{\sigma}^{\mathrm{o}}
$$

The components of the positive definite fourth-rank tensor $\mathbf{T}$ read

$$
T_{i j k l}=T_{1} \delta_{i j} \delta_{k l}+T_{2}\left(\delta_{i k} \delta_{j l}+\delta_{i l} \delta_{j k}\right), \quad i, j, k, l=1,2
$$

with

$$
\begin{aligned}
2 T_{1}+2 T_{2} & =\frac{1}{3}\left(1-2 v_{A}^{*}\right)^{2}+\sum_{q=1}^{2} \frac{4\left(1-2 v_{0}\right)^{2} \phi_{q}}{\left(\alpha_{q}+\beta_{q}\right)^{2}}, \\
T_{2} & =\frac{1}{2}+\frac{1}{6} \sum_{q=1}^{2} \frac{\left.\left(8 v_{A}^{*} 2-8 v_{A}^{*}+5\right)\right) \phi_{q}}{\beta_{q}^{2}}
\end{aligned}
$$


in which the (current) volume fraction of the $q$-phase is defined as $\phi_{q} \equiv$ $\pi a^{2}\left(N_{q} / A\right)$,

$$
\begin{aligned}
& \alpha_{1}=\left(4 v_{0}-1\right)+\frac{\left(\lambda_{0} \mu_{1}-\lambda_{1} \mu_{0}\right)\left[8\left(1-v_{0}\right)\right]}{2\left(\mu_{1}-\mu_{0}\right)\left(\lambda_{1}-\lambda_{0}+\mu_{1}-\mu_{0}\right)}, \quad \alpha_{2}=\left(4 v_{0}-1\right) \\
& \beta_{1}=\left(3-4 v_{0}\right)+4\left(1-v_{0}\right) \frac{\mu_{0}}{\left(\mu_{1}-\mu_{0}\right)}, \quad \beta_{2}=-1
\end{aligned}
$$

and $v_{A}^{*}$ is effective axial Poisson's ratio.

The ensemble-averaged current stress norm at a matrix point can also be expressed in terms of the macroscopic stress $\overline{\boldsymbol{\sigma}}$. Following Ju and Chen (1994c), the relation between the far-field stress $\sigma^{\circ}$ and macroscopic stress $\overline{\boldsymbol{\sigma}}$ can be expressed as

$$
\boldsymbol{\sigma}^{\mathrm{o}}=\mathbf{P}: \overline{\boldsymbol{\sigma}}
$$

where the components of $\mathbf{P}$ are

$$
P_{i j k l}=P_{1} \delta_{i j} \delta_{k l}+P_{2}\left(\delta_{i k} \delta_{j l}+\delta_{i l} \delta_{j k}\right), \quad i, j, k, l=1,2
$$

with

$$
\begin{aligned}
P_{1} & =-\frac{m_{5}}{4 m_{6}\left(m_{5}+m_{6}\right)}, \quad P_{2}=\frac{1}{4 m_{6}} \\
m_{5} & =\sum_{q=1}^{2} \frac{\left[-4 \alpha_{q}\left(-1+v_{0}\right)+\beta_{q}\left(-5+8\left(3-2 v_{0}\right) \nu_{0}\right)\right] \phi_{q}}{8 \beta_{q}\left(\alpha_{q}+\beta_{q}\right)\left(-1+v_{0}\right)}, \quad m_{6}=\frac{1}{2}+\sum_{q=1}^{2} \frac{\phi_{q}}{2 \beta_{q}}
\end{aligned}
$$

Combining Equations (39) and (44), the ensemble-averaged current stress norm at a matrix point reads:

$$
\langle H\rangle_{m}(\mathbf{x})=\overline{\boldsymbol{\sigma}}: \overline{\mathbf{T}}: \overline{\boldsymbol{\sigma}}
$$

where the positive definite fourth-rank tensor $\overline{\mathbf{T}}$ is defined as

$$
\overline{\mathbf{T}} \equiv \mathbf{P}^{\mathrm{T}}: \mathbf{T}: \mathbf{P}
$$

and can be shown to be

$$
\bar{T}_{i j k l}=\bar{T}_{1} \delta_{i j} \delta_{k l}+\bar{T}_{2}\left(\delta_{i k} \delta_{j l}+\delta_{i l} \delta_{j k}\right), \quad i, j, k, l=1,2
$$


where

$$
\bar{T}_{1}=4\left(P_{1}^{2} T_{1}+2 P_{1} P_{2} T_{1}+P_{2}^{2} T_{1}+P_{1}^{2} T_{2}+2 P_{1} P_{2} T_{2}\right), \quad \bar{T}_{2}=4 P_{2}^{2} T_{2}
$$

The ensemble-averaged 'current stress norm' for any point $\mathbf{x}$ in a threephase damaged fiber composite can be defined as:

$$
\sqrt{\langle H\rangle(\mathbf{x})}=\left(1-\phi_{1}\right) \sqrt{\overline{\boldsymbol{\sigma}}: \overline{\mathbf{T}}: \overline{\boldsymbol{\sigma}}}
$$

where $\phi_{1}$ is the current fiber volume fraction. Therefore, the effective yield function for the three-phase FRDMCs can be proposed as

$$
\bar{F}=\left(1-\phi_{1}\right) \sqrt{\overline{\boldsymbol{\sigma}}: \overline{\mathbf{T}}: \overline{\boldsymbol{\sigma}}}-K\left(\bar{e}^{p}\right)
$$

with the isotropic hardening function $K\left(\bar{e}^{p}\right)$ for the three-phase composite materials. Our formulation can accommodate kinematic hardening function as well, but isotropic hardening function is used here for simplicity. In addition, we assume that the overall flow rule for the composite materials is associative. Therefore, the effective ensemble-averaged plastic strain rate for the FRDMCs can be expressed as

$$
\dot{\overline{\boldsymbol{\varepsilon}}}^{p}=\dot{\lambda} \frac{\partial \bar{F}}{\partial \bar{\sigma}}=\left(1-\phi_{1}\right) \dot{\bar{\lambda}} \frac{\overline{\mathbf{T}}: \overline{\boldsymbol{\sigma}}}{\sqrt{\overline{\boldsymbol{\sigma}}: \overline{\mathbf{T}}: \overline{\boldsymbol{\sigma}}}}
$$

where $\dot{\lambda}$ denotes the plastic consistency parameter. In general, the overall flow rule of the composite materials may become non-associative when fibers, cracks, and voids exist according to dislocation dynamics analysis. Extension to the non-associative flow rule can be constructed in a similar fashion, but involving both the normal and tangential flow directions.

Inspired by the structure of the micromechanically derived stress norm, the effective equivalent plastic strain rate for the composites is defined as

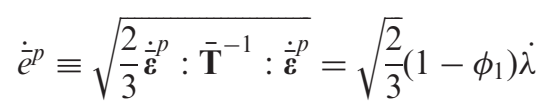

The $\dot{\lambda}$ and the yield function $\bar{F}$ must obey the Kuhn-Tucker loading/ unloading conditions:

$$
\dot{\lambda} \geq 0, \quad \bar{F} \leq 0, \quad \dot{\lambda} \bar{F}=0, \quad \dot{\lambda} \dot{\bar{F}}=0
$$


The ensemble-averaged yield function in Equation (53), the averaged plastic flow rule in Equation (54), the equivalent plastic strain rate in Equation (55), and the Kuhn-Tucker conditions completely characterize the effective plasticity formulation for the composite materials with the isotropic hardening function $K\left(\bar{e}^{p}\right)$. Here, the power-law type isotropic hardening function is used as an example:

$$
K\left(\bar{e}^{p}\right)=\sqrt{\frac{2}{3}}\left\{\overline{\boldsymbol{\sigma}}_{y}+h\left(\bar{e}^{p}\right)^{q}\right\}
$$

where $\bar{\sigma}_{y}$ is the initial yield stress, and $h$ and $q$ signify the linear and exponential isotropic hardening parameters, respectively, for the three-phase composites.

\section{EVOLUTIONARY INTERFACIAL DEBONDING}

\section{Probabilistic Micromechanics}

The progressive, complete interfacial debonding may occur under increasing deformations, and thus influence the overall behavior of composites. After the interfacial debonding between fibers and the matrix, the debonded fibers lose the load-carrying capacity and are assumed to become voids. Within the context of the first-order approximation, the stresses inside fibers would be uniform. For convenience, following Tohgo and Weng (1994) and Zhao and Weng (1995,1996,1997), we employ the average internal stresses of a fiber as the controlling factor. The probability of complete fiber debonding is modeled as a two-parameter Weibull process. Assuming that the Weibull (1951) statistics governs, we express the cumulative probability distribution function of fiber debonding, $P_{d}$, for the uniaxial tensile loading (in the 11-direction) as:

$$
P_{d}\left[\left(\bar{\sigma}_{11}\right)_{1}\right]=1-\exp \left[-\left(\frac{\left(\bar{\sigma}_{11}\right)_{1}}{S_{0}}\right)^{M}\right]
$$

where $\left(\bar{\sigma}_{11}\right)_{1}$ is the average internal stresses of fibers (phase1) in the 11direction, the subscript $(\cdot)_{1}$ denotes the fiber phase, and $S_{0}$ and $M$ are the Weibull parameters representing the average interfacial strength and debonding evolution rate, respectively. When one decreases $S_{0}$ or increases $M$ values, it would accelerate the damage evolution rate. 
Therefore, the current void volume fraction $\phi_{2}$ at a given level of $\left(\bar{\sigma}_{11}\right)_{1}$ is given by

$$
\phi_{2}=\phi P_{d}\left[\left(\bar{\sigma}_{11}\right)_{1}\right]=\phi\left\{1-\exp \left[-\left(\frac{\left(\bar{\sigma}_{11}\right)_{1}}{S_{0}}\right)^{M}\right]\right\}
$$

where $\phi$ is the initial fiber volume fraction. Under the plane-strain condition, for the biaxial tensile loading, $\left(\bar{\sigma}_{11}\right)_{1}$ in Equations (58) and (59) is replaced by $\left[\left(\bar{\sigma}_{11}\right)_{1}-v_{T}^{*}\left(\bar{\sigma}_{22}\right)_{1}\right]$ if $\left(\bar{\sigma}_{11}\right)_{1} \geq\left(\bar{\sigma}_{22}\right)_{1}$, or by $\left[\left(\bar{\sigma}_{22}\right)_{1}-v_{T}^{*}\left(\bar{\sigma}_{11}\right)_{1}\right]$ if $\left(\bar{\sigma}_{11}\right)_{1} \leq\left(\bar{\sigma}_{22}\right)_{1}$.

\section{Internal Stresses of Fibers}

In order to determine the probability of fiber debonding, we need to derive the internal stresses of fibers. According to the Eshelby's equivalence principle, the perturbed strain field $\boldsymbol{\varepsilon}^{\prime}(\mathbf{x})$ induced by inhomogeneities can be related to specified eigenstrain $\boldsymbol{\varepsilon}^{*}(\mathbf{x})$ by replacing the inhomogeneities with the matrix material. That is, for the domain of the $q$-phase with elastic stiffness tensor $\mathbf{C}_{q}$, we have

$$
\boldsymbol{\sigma}_{q}=\mathbf{C}_{q}:\left[\varepsilon^{\mathrm{o}}+\boldsymbol{\varepsilon}^{\prime}(\mathbf{x})\right]=\mathbf{C}_{0}:\left[\varepsilon^{\mathrm{o}}+\boldsymbol{\varepsilon}^{\prime}(\mathbf{x})-\boldsymbol{\varepsilon}^{*}(\mathbf{x})\right]
$$

where $\boldsymbol{\sigma}_{q}$ is the total local stresses for the $q$-phase. The ensemble-averaged strain for the three-phase composites takes the form (Ju and Chen, 1994a, b)

$$
\overline{\boldsymbol{\varepsilon}}=\boldsymbol{\varepsilon}^{\mathrm{o}}+\sum_{q=1}^{2} \phi_{q} \mathbf{S}: \overline{\boldsymbol{\varepsilon}}_{q}^{*}
$$

where the 'first-order' solution $\boldsymbol{\varepsilon}_{q}^{* 0}$ of the eigenstrain $\boldsymbol{\varepsilon}_{q}^{*}$ has previously been given in Equation (35). Substituting Equation (35) into Equation (61), we arrive at

$$
\overline{\boldsymbol{\varepsilon}}=\left[\mathbf{I}-\sum_{q=1}^{2} \boldsymbol{\phi}_{q} \mathbf{S} \bullet\left(\mathbf{A}_{q}+\mathbf{S}\right)^{-1}\right]: \boldsymbol{\varepsilon}^{\mathrm{o}}
$$

The average stress tensor for $q$-phase is defined as

$$
\overline{\boldsymbol{\sigma}}_{q} \equiv \frac{1}{\mathbf{A}_{q}} \int_{A_{q}} \boldsymbol{\sigma}_{q}(\mathbf{x}) \mathrm{d} \mathbf{x}=\frac{1}{A_{q}}\left\{\int_{A q} \mathbf{C}_{q}:\left[\varepsilon^{\mathrm{o}}+\boldsymbol{\varepsilon}^{\prime}(\mathbf{x})\right] \mathrm{d} x\right\} \equiv \mathbf{C}_{q}:\left[\boldsymbol{\varepsilon}^{\mathrm{o}}+\overline{\boldsymbol{\varepsilon}}_{q}^{\prime}\right]
$$


where $\overline{\boldsymbol{\varepsilon}}_{q}^{\prime}$ can be recast as

$$
\overline{\boldsymbol{\varepsilon}}_{q}^{\prime}=\mathbf{S}:\left[\frac{1}{A_{q}} \int_{A_{q}} \overline{\boldsymbol{\varepsilon}}^{*}\left(\mathbf{x}^{\prime}\right) \mathrm{d} \mathbf{x}^{\prime}\right]=\mathbf{S}: \overline{\boldsymbol{\varepsilon}}_{q}^{*}
$$

By combining Equation (35) and Equations (62) and (64), we arrive at

$$
\overline{\boldsymbol{\sigma}}_{q}=\mathbf{C}_{q} \bullet\left[\mathbf{I}-\mathbf{S} \bullet\left(\mathbf{A}_{q}+\mathbf{S}\right)^{-1}\right] \bullet\left[\mathbf{I}-\sum_{q=1}^{2} \phi_{q} \mathbf{S} \bullet\left(\mathbf{A}_{q}+\mathbf{S}\right)^{-1}\right]^{-1}: \overline{\boldsymbol{\varepsilon}}
$$

The above derivation is equally applicable to ensemble-averaged quantities by applying the ensemble averaging operator $\langle\cdot\rangle$ to $\overline{\boldsymbol{\sigma}}_{q}$ and $\overline{\boldsymbol{\varepsilon}}$. Therefore, the averaged internal stresses of fibers can be expressed as $(q=1)$

$$
\overline{\boldsymbol{\sigma}}_{1}=\mathbf{C}_{1} \bullet\left[\mathbf{I}-\mathbf{S} \bullet\left(\mathbf{A}_{1}+\mathbf{S}\right)^{-1}\right] \bullet\left[\mathbf{I}-\sum_{q=1}^{2} \phi_{q} \mathbf{S} \bullet\left(\mathbf{A}_{q}+\mathbf{S}\right)^{-1}\right]^{-1}: \overline{\boldsymbol{\varepsilon}} \equiv \mathbf{U}: \overline{\boldsymbol{\varepsilon}}
$$

By carrying out the lengthy algebra, the components of the positive definite fourth-rank tensor $\mathbf{U}$ are explicitly given by

$$
U_{i j k l}=U_{1} \delta_{i j} \delta_{k l}+U_{2}\left(\delta_{i k} \delta_{j l}+\delta_{i l} \delta_{j k}\right), \quad i, j, k, l=1,2
$$

where

$$
U_{1}=2\left(\lambda_{1} u_{1}^{\prime}+\lambda_{1} u_{2}^{\prime}+u_{1}^{\prime} \mu_{1}\right), \quad U_{2}=4 \mu_{1} \bar{\mu} \hat{\mu}
$$

and

$$
\begin{aligned}
& u_{1}^{\prime}=2(\bar{\lambda} \hat{\lambda}+\bar{\lambda} \hat{\mu}+\hat{\lambda} \bar{\mu}), \quad u_{2}^{\prime}=2 \bar{\mu} \hat{\mu}, \quad \hat{\lambda}=-\frac{w_{1}}{4 w_{2}\left(w_{1}+w_{2}\right)}, \quad \hat{\mu}=\frac{1}{4 w_{2}} \\
& w_{1}=-\left(D_{5}+E_{5}\right), \quad w_{2}=\frac{1}{2}-\left(D_{6}+E_{6}\right), \quad E_{5}=\frac{4\left(\alpha_{2}+2 \beta_{2}\right) v_{0}-\left(3 \alpha_{2}+2 \beta_{2}\right)}{4 \beta_{2}\left(\alpha_{2}+\beta_{2}\right)} \\
& E_{6}=\frac{3-4 v_{0}}{2 \beta_{2}}, \quad \bar{\lambda}=-D_{5}, \quad \bar{\mu}=\frac{1}{2}-D_{6}, \quad D_{5}=\frac{4\left(\alpha_{1}+2 \beta_{1}\right) v_{0}-\left(3 \alpha_{1}+2 \beta_{1}\right)}{4 \beta_{1}\left(\alpha_{1}+\beta_{1}\right)} \\
& D_{6}=\frac{3-4 v_{0}}{2 \beta_{1}}
\end{aligned}
$$


In the case of tensile loading, the averaged internal stresses of fibers can be obtained as:

$$
\begin{aligned}
& \left(\overline{\boldsymbol{\sigma}}_{11}\right)_{1}=\left(U_{1}+2 U_{2}\right) \overline{\boldsymbol{\varepsilon}}_{11}+U_{1} \overline{\boldsymbol{\varepsilon}}_{22} \\
& \left(\overline{\boldsymbol{\sigma}}_{22}\right)_{1}=U_{1} \overline{\boldsymbol{\varepsilon}}_{11}+\left(U_{1}+2 U_{2}\right) \overline{\boldsymbol{\varepsilon}}_{22}
\end{aligned}
$$

where $\bar{\varepsilon}_{11}$ and $\bar{\varepsilon}_{22}$ are the ensemble-averaged strains in the 11- and 22directions, respectively.

\section{ELASTOPLASTIC DAMAGE STRESS-STRAIN RESPONSES FOR COMPOSITES}

To illustrate the proposed micromechanics-based effective elastoplastic damage formulation for FRDMCs, let us consider the examples of the uniaxial and biaxial tensile loading under the plane-strain condition in this section.

\section{Elastoplastic Damage Stress-Strain Relation under Uniaxial Tensile Loading}

The applied macroscopic stress $\overline{\boldsymbol{\sigma}}$ can be written as

$$
\bar{\sigma}_{11}>0, \quad \bar{\sigma}_{33}=v_{A}^{*} \bar{\sigma}_{11}, \quad \text { all other } \bar{\sigma}_{i j}=0 .
$$

With the isotropic hardening law described by Equation (57), the overall yield function becomes

$$
\bar{F}\left(\overline{\boldsymbol{\sigma}}, \bar{e}^{p}\right)=\left(1-\phi_{1}\right) \sqrt{\overline{\boldsymbol{\sigma}}: \overline{\mathbf{T}}: \overline{\boldsymbol{\sigma}}}-\sqrt{\frac{2}{3}}\left\{\bar{\sigma}_{y}+h\left(\bar{e}^{p}\right)^{q}\right\}
$$

Substituting Equation (74) into (75), the effective yield function for the uniaxial loading is obtained as

$$
\bar{F}\left(\bar{\sigma}_{11}, \bar{e}^{p}\right)=\left(1-\phi_{1}\right) \sqrt{\left(\bar{T}_{1}+2 \bar{T}_{2}\right)} \bar{\sigma}_{11}-\sqrt{\frac{2}{3}}\left\{\bar{\sigma}_{y}+h\left(\bar{e}^{p}\right)^{q}\right\}
$$

The macroscopic incremental plastic strain defined by Equation (54) then reads

$$
\Delta \overline{\boldsymbol{\varepsilon}}^{p}=\left(1-\phi_{1}\right) \frac{\Delta \lambda}{\sqrt{\left(\bar{T}_{1}+2 \bar{T}_{2}\right)}}\left[\begin{array}{cc}
\bar{T}_{1}+2 \bar{T}_{2} & 0 \\
0 & \bar{T}_{1}
\end{array}\right]
$$


for any stress beyond the initial yielding, where $\Delta \lambda$ is the incremental plastic consistency parameter. Similarly, the incremental equivalent plastic strain can be expressed as:

$$
\Delta \bar{e}^{p}=\sqrt{\frac{2}{3}}\left(1-\phi_{1}\right) \Delta \lambda
$$

From the linear elasticity theory, the macroscopic incremental elastic strain takes the form:

$$
\Delta \overline{\boldsymbol{\varepsilon}}^{e}=\left[\begin{array}{cc}
E_{A}^{*}-E_{T}^{*} \nu_{A}^{* 2} & 0 \\
0 & -E_{A}^{*} \nu_{T}^{*}-E_{T}^{*} \nu_{A}^{* 2}
\end{array}\right] \frac{\Delta \bar{\sigma}_{11}}{E_{T}^{*} E_{A}^{*}}
$$

For the monotonic plane-strain uniaxial loading, the overall incremental macroscopic stress-strain relation can be obtained by summing Equations (77) and (79) as follows:

$$
\begin{aligned}
\Delta \overline{\boldsymbol{\varepsilon}}= & {\left[\begin{array}{cc}
E_{A}^{*}-E_{T}^{*} v_{A}^{* 2} & 0 \\
0 & -E_{A}^{*} \nu_{T}^{*}-E_{T}^{*} \nu_{A}^{* 2}
\end{array}\right] \frac{\Delta \bar{\sigma}_{11}}{E_{T}^{*} E_{A}^{*}} } \\
& +\left(1-\phi_{1}\right) \frac{\Delta \lambda}{\sqrt{\left(\bar{T}_{1}+2 \bar{T}_{2}\right)}}\left[\begin{array}{cc}
\bar{T}_{1}+2 \bar{T}_{2} & 0 \\
0 & \bar{T}_{1}
\end{array}\right]
\end{aligned}
$$

where the positive parameter $\Delta \lambda$ is solved from the nonlinear equation obtained by enforcing the plastic consistency condition $\bar{F}=0$ :

$$
\left(1-\phi_{1}\right) \sqrt{\left(\bar{T}_{1}+2 \bar{T}_{2}\right)}\left(\bar{\sigma}_{11}\right)_{n+1}=\sqrt{\frac{2}{3}}\left\{\bar{\sigma}_{y}+h\left[\bar{e}_{n}^{P}+\Delta \bar{e}_{n+1}^{P}\right]^{q}\right\}
$$

Here, $\left(\bar{\sigma}_{11}\right)_{n+1}$ and $\bar{e}_{n+1}^{P}$ are the prescribed macroscopic stress along the 11-direction and the incremental equivalent plastic strain at the current time step, respectively. Moreover, $\bar{e}_{n}^{P}$ is the equivalent plastic strain at the previous time step. Equations (78) and (81) then lead to:

$$
\left(1-\phi_{1}\right) \sqrt{\left(\bar{T}_{1}+2 \bar{T}_{2}\right)}\left(\bar{\sigma}_{11}\right)_{n+1}=\sqrt{\frac{2}{3}}\left\{\bar{\sigma}_{y}+h\left[\bar{e}_{n}^{P}+\sqrt{\frac{2}{3}}\left(1-\phi_{1}\right) \Delta \lambda\right]^{q}\right\}
$$

Therefore, the expression for $\Delta \lambda$ becomes

$$
\Delta \lambda=\frac{1}{\sqrt{2 / 3}\left(1-\phi_{1}\right)}\left\{\left[\frac{\left(1-\phi_{1}\right) \sqrt{3 / 2}\left(\bar{T}_{1}+2 \bar{T}_{2}\right)\left(\bar{\sigma}_{11}\right)_{n+1}-\bar{\sigma}_{y}}{h}\right]^{1 / q}-\bar{e}_{n}^{p}\right\}
$$


Due to the evolutionary fiber debonding, both the fiber volume fraction $\phi_{1}$ and the void volume fraction $\phi_{2}$ are functions of stresses. Further, effective elastic moduli and the plastic parameters of $\bar{T}_{1}$ and $\bar{T}_{2}$ are functions of the fiber and void volume fractions.

\section{Elastoplastic Damage Stress-Strain Relation under Biaxial Tensile Loading}

The applied macroscopic stress $\overline{\boldsymbol{\sigma}}$ can be rephrased as

$$
\bar{\sigma}_{11}>0, \quad \bar{\sigma}_{22}=R \bar{\sigma}_{11}, \quad \bar{\sigma}_{33}=v_{A}^{*}\left(\bar{\sigma}_{11}+\bar{\sigma}_{22}\right), \quad \text { all other } \bar{\sigma}_{i j}=0 .
$$

Here, $R$ is a parameter of loading stress ratio. Specifically, if $R=0$, the biaxial loading will reduce to the uniaxial loading. Substituting Equation (84) into (75), the effective yield function for the case of biaxial tensile loading becomes

$$
\bar{F}\left(\bar{\sigma}_{11}, \bar{e}^{p}\right)=\left(1-\phi_{1}\right) \Phi(R) \bar{\sigma}_{11}-\sqrt{\frac{2}{3}}\left\{\bar{\sigma}_{y}+h\left(\bar{e}^{p}\right)^{q}\right\}
$$

where

$$
\Phi(R)=\sqrt{\bar{T}_{1}(1+R)^{2}+2 \bar{T}_{2}\left(1+R^{2}\right)}
$$

The macroscopic incremental plastic strain defined by Equation (54) becomes

$$
\Delta \overline{\boldsymbol{\varepsilon}}^{p}=\left(1-\phi_{1}\right) \frac{\Delta \lambda}{\Phi(R)}\left[\begin{array}{cc}
(1+R) \bar{T}_{1}+2 \bar{T}_{2} & 0 \\
0 & (1+R) \bar{T}_{1}+2 R \bar{T}_{2}
\end{array}\right]
$$

for any stress beyond the initial yielding. Similarly, the incremental equivalent plastic strain can be written as

$$
\Delta \bar{e}^{p}=\sqrt{\frac{2}{3}}\left(1-\phi_{1}\right) \Delta \lambda
$$

The macroscopic incremental elastic strain takes the form

$$
\Delta \overline{\boldsymbol{\varepsilon}}^{e}=\left[\begin{array}{cc}
E_{A}^{*}\left(1-v_{T}^{*} R\right)-E_{T}^{*} v_{A}^{* 2}(1+R) & 0 \\
0 & \left(R-v_{T}^{*}\right) E_{A}^{*}-E_{T}^{*} v_{A}^{* 2}(1+R)
\end{array}\right] \frac{\Delta \bar{\sigma}_{11}}{E_{T}^{*} E_{A}^{*}}
$$


For the monotonic plane-strain biaxial tensile loading, the overall incremental macroscopic stress-strain relation can be obtained by summing Equations (87) and (89):

$$
\begin{aligned}
\Delta \bar{\varepsilon}= & {\left[\begin{array}{cc}
E_{A}^{*}\left(1-v_{T}^{*} R\right)-E_{T}^{*} v_{A}^{* 2}(1+R) & 0 \\
0 & \left(R-v_{T}^{*}\right) E_{A}^{*}-E_{T}^{*} v_{A}^{* 2}(1+R)
\end{array}\right] \frac{\Delta \bar{\sigma}_{11}}{E_{T}^{*} E_{A}^{*}} } \\
& +\left(1-\phi_{1}\right) \frac{\Delta \lambda}{\Phi(R)}\left[\begin{array}{cc}
(1+R) \bar{T}_{1}+2 \bar{T}_{2} & 0 \\
0 & (1+R) \bar{T}_{1}+2 R \bar{T}_{2}
\end{array}\right]
\end{aligned}
$$

where the positive parameter $\Delta \lambda$ is solved from the nonlinear equation obtained by enforcing the plastic consistency condition $\bar{F}=0$ :

$$
\left(1-\phi_{1}\right) \Phi(R)\left(\bar{\sigma}_{11}\right)_{n+1}=\sqrt{\frac{2}{3}}\left\{\bar{\sigma}_{y}+h\left[\bar{e}_{n}^{P}+\Delta \bar{e}_{n+1}^{P}\right]^{q}\right\}
$$

Again, $\left(\bar{\sigma}_{11}\right)_{n+1}$ and $\Delta \bar{e}_{n+1}^{P}$ are the prescribed macroscopic stress along the 11-direction and the incremental equivalent plastic strain at the current time step, respectively. Here, $\bar{e}_{n}^{P}$ is the equivalent plastic strain at the previous load step. Equations (88) and (91) then result in

$$
\left(1-\phi_{1}\right) \Phi(R)\left(\bar{\sigma}_{11}\right)_{n+1}=\sqrt{\frac{2}{3}}\left\{\bar{\sigma}_{y}+h\left[\bar{e}_{n}^{P}+\sqrt{\frac{2}{3}}\left(1-\phi_{1}\right) \Delta \lambda\right]^{q}\right\}
$$

Therefore, the expression for $\Delta \lambda$ reads

$$
\Delta \lambda=\frac{1}{\sqrt{2 / 3}\left(1-\phi_{1}\right)}\left\{\left[\frac{\left(1-\phi_{1}\right) \sqrt{3 / 2} \Phi(R)\left(\bar{\sigma}_{11}\right)_{n+1}-\bar{\sigma}_{y}}{h}\right]^{1 / q}-\bar{e}_{n}^{p}\right\}
$$

\section{COMPUTATIONAL STRAIN-DRIVEN INTEGRATION ALGORITHM}

In this section, we present the efficient strain-driven algorithm where the macroscopic stress history $\overline{\boldsymbol{\sigma}}$ is to be uniquely determined by the given macroscopic strain history $\overline{\boldsymbol{\varepsilon}}$. We determine the unknown state of a local point $\left(\overline{\boldsymbol{\sigma}}_{n+1}, \overline{\boldsymbol{\varepsilon}}_{n+1}^{p}, \bar{e}_{n+1}^{p}\right)$ at the end of the time step $t=t_{n+1}$ given the known state from the previous time step $\left\{\overline{\boldsymbol{\varepsilon}}_{n}, \overline{\boldsymbol{\varepsilon}}_{n}^{p}, e_{n}^{p}\right\}$ at $t=t_{n}$.

For convenience, Table 1 and Table 2 summarize the micromechanical iterative computational strain-driven algorithm for the elastoplastic damage behavior of ductile matrix composites accounting for interfacial debonding 
Table 1. Micromechanical iterative algorithm for the evolutionary damage formulation.

(i) Estimate the model parameters:

1. Plastic material parameters: $\bar{\sigma}_{y}, h, q$;

2. Weibull parameters: $S_{0}, M$

(ii) Compute elastic coefficients: $\alpha_{\mathrm{q}}, \beta_{\mathrm{q}}, \mathrm{a}_{\mathrm{q}}, \mathrm{b}_{\mathrm{q}}(q=1,2)$

(iii) Initialize: Set $\mathrm{z}=0$; $\left(\phi_{1}\right)_{n+1}^{(0)}=\left(\phi_{1}\right)_{n},\left(\phi_{2}\right)_{n+1}^{(0)}=\left(\phi_{2}\right)_{n}$

(iv) Compute:

Coefficients: $\mathbf{T}_{n+1}^{(z)}, \mathbf{T}_{n+1}^{(z)}, \mathbf{U}_{n+1}^{(z)}$

Effective Moduli: $\left(E_{A}^{*}\right)_{n+1}^{(z)},\left(v_{A}^{*}\right)_{n+1}^{(z)},\left(E_{T}^{*}\right)_{n+1}^{(z)},\left(v_{T}^{*}\right)_{n+1}^{(z)}$

(v) Compute internal stresses of fibers:

$$
\begin{aligned}
& {\left[\left(\bar{\sigma}_{11}\right)_{1}\right]_{n+1}^{(z)}=\left[\left(U_{1}\right)_{n+1}^{(z)}+2\left(U_{2}\right)_{n+1}^{(z)}\right]\left(\bar{\varepsilon}_{11}\right)_{n+1}+\left(U_{1}\right)_{n+1}^{(z)}\left(\bar{\varepsilon}_{22}\right)_{n+1}} \\
& {\left[\left(\bar{\sigma}_{22}\right)_{1}\right]_{n+1}^{(z)}=\left(U_{1}\right)_{n+1}^{(z)}\left(\bar{\varepsilon}_{11}\right)_{n+1}+\left[\left(U_{1}\right)_{n+1}^{(z)}+2\left(U_{2}\right)_{n+1}^{(z)}\right]\left(\bar{\varepsilon}_{22}\right)_{n+1}}
\end{aligned}
$$

(vi) Compute the Weibull probability distribution function:

$$
P_{\mathrm{d}}\left[\left(\bar{\sigma}_{11}\right)_{1}\right]_{n+1}^{(z)}=1-\exp \left[-\left(\frac{\left[\left(\bar{\sigma}_{11}\right)_{1}\right]_{n+1}^{(z)}}{S_{0}}\right)^{M}\right]
$$

(vii) Compute volume fractions:

$$
\begin{aligned}
& \left(\phi_{2}\right)_{n+1}^{(z)}=\phi P_{d}\left\{\left[\left(\bar{\sigma}_{11}\right)_{1}\right]_{n+1}^{(z)}\right\}=\phi\left\{1-\exp \left[-\left(\frac{\left[\left(\bar{\sigma}_{11}\right)_{1}\right]_{n+1}^{(z)}}{S_{0}}\right)^{M}\right]\right\}, \\
& \left(\phi_{1}\right)_{n+1}^{(z)}=\phi-\left(\phi_{2}\right)_{n+1}^{(z)}
\end{aligned}
$$

(viii) Perform convergence check:

If $\left|\left(\phi_{1}\right)_{n+1}^{(z)}-\left(\phi_{1}\right)_{n+1}^{(z-1)}\right| \leq$ TOL (e.g., 10 $\left.{ }^{-8}\right)$, then update quantities (iv) $\sim($ vii)

$T_{n+1}=T_{n+1}^{(z)}, \ldots,\left(E_{A}^{*}\right)_{n+1}=\left(E_{A}^{*}\right)_{n+1}^{(z)} ;\left[\left(\bar{\sigma}_{\mathrm{ii}}\right)_{1}\right]_{n+1}=\left[\left(\bar{\sigma}_{\mathrm{ii}}\right)_{1}\right]_{n+1}^{(z)},(i=1,2) ;$

$\left(\phi_{1}\right)_{n+1}=\left(\phi_{1}\right)_{n+1}^{(z)} ;\left(\phi_{2}\right)_{n+1}=\left(\phi_{2}\right)_{n+1}^{(z)} ;$ GO TO Step (ix) in Table 2.

Otherwise: SET $z=z+1$; GO TO (iv).

evolution. In particular, Table 1 renders a step-by-step flow chart of the computational procedure to determine the current fiber volume fractions $\left(\phi_{1}\right)_{n+1}$ in accordance with the evolutionary interfacial debonding. Further, Table 2 provides a detailed iterative return mapping algorithm consistent with the current formulation.

\section{Numerical Simulations and Experimental Comparison}

The experimental studies to characterize damage evolutions in FRDMCs have been rather limited in the literature. To assess the predictive capability 
Table 2. Return mapping algorithm.

(ix) Return mapping algorithm:

(a) Initialize: Set $I=0$; $\left(\bar{\varepsilon}_{n+1}^{p}\right)^{(0)}=\bar{\varepsilon}_{n}^{p},\left(\bar{e}_{n+1}^{p}\right)=\bar{e}_{n}^{p}$ (for local Newton iteration)

(b) Compute: $\boldsymbol{\sigma}_{n}=\mathbf{C}_{n}:\left[\bar{\varepsilon}_{n}-\bar{\varepsilon}_{n}^{p}\right]$

(c) Compute the elastic predictor:

Trial elastic state: $\overline{\boldsymbol{\sigma}}_{\mathrm{n}+1}^{\mathrm{tr}}=\overline{\boldsymbol{\sigma}}_{n}+\mathbf{C}_{n}: \Delta \overline{\boldsymbol{\varepsilon}}_{\mathrm{n}+1}$

Compute: $\bar{\varepsilon}_{n+1}=\bar{\varepsilon}_{n}+\Delta \bar{\varepsilon}_{n+1}$;

$\bar{F}_{n+1}^{\mathrm{tr}}\left(\overline{\boldsymbol{\sigma}}_{n+1}^{\mathrm{tr}}, \bar{e}_{n}^{p}\right)=\left(1-\phi_{1}\right) \sqrt{\overline{\boldsymbol{\sigma}}_{n+1}^{\mathrm{tr}}: \mathbf{T}: \overline{\boldsymbol{\sigma}}_{n+1}^{\mathrm{tr}}}-K\left(\bar{e}^{p}\right)$

(d) Check whether plastic loading is active:

If $\bar{F}_{n+1}^{\mathrm{tr}} \leq \mathrm{TOL}$ (elastic step; e.g., TOL $=10^{-8}$ ):

$\hat{\xi}_{n+1}=0$; SET $\overline{\boldsymbol{\sigma}}_{n+1}=\overline{\boldsymbol{\sigma}}_{n+1}^{\mathrm{tr}}, \overline{\mathrm{e}}_{n+1}^{p}=\bar{e}_{n}^{p}$; GO TO (iv) in Table 1.

Otherwise (plastic step): GO TO (e)

(e) Perform plastic correction; return mapping algorithm:

Solve nonlinear scalar equation for $\hat{\xi}_{\mathrm{n}+1}$ : use local Newton iteration

$\bar{F}_{n+1}\left(\hat{\xi}_{n+1}^{(l)}\right)=\left(1-\left(\phi_{1}\right)_{n+1}\right) \sqrt{\overline{\boldsymbol{\sigma}}_{n+1}^{(l)}: \mathbf{T}: \overline{\boldsymbol{\sigma}}_{n+1}^{(l)}}-K\left[\left(\bar{e}_{n+1}^{p}\right)^{(l)}\right]=0$

(f) Perform convergence check:

If $\left|\bar{F}_{n+1}\left(\hat{\xi}_{n+1}^{(l)}\right)\right| \leq \mathrm{TOL}\left(\right.$ e.g., $\left.10^{-8}\right)$, then update

$\hat{\xi}_{n+1}=\hat{\xi}_{n+1}^{(l)} ; \overline{\boldsymbol{\sigma}}_{n+1}=\overline{\boldsymbol{\sigma}}_{n+1}^{(l)}, \bar{e}_{n+1}^{p}=\left(\bar{e}_{n+1}^{p}\right)^{(l)}, \bar{\varepsilon}_{n+1}^{p}=\left(\bar{\varepsilon}_{n+1}^{p}\right)^{(l)} ;$ EXIT.

Otherwise: $\mathrm{SET} I=I+1$

Compute derivative of $\bar{F}_{n+1}\left[\left(\hat{\xi}_{n+1}^{(l)}\right)\right] \rightarrow D \bar{F}\left[\left(\hat{\xi}_{n+1}^{(l)}\right)\right]$

$\left(\hat{\xi}_{n+1}^{(l+1)}\right)=\left(\hat{\xi}_{n+1}^{(l)}\right)-\frac{\bar{F}\left[\left(\hat{\xi}_{n+1}^{(l)}\right)\right]}{D \bar{F}\left[\left(\hat{\xi}_{n+1}^{(l)}\right)\right]} ;$ GO TO (e).

of the present framework, comparisons are made in this section between the present predictions and the experimental data as quoted by Nimmer et al. (1991). The experimental data were observed at 427,315 , and $23^{\circ} \mathrm{C}$ for the $\mathrm{SiC} / \mathrm{Ti}-6 \mathrm{Al}-4 \mathrm{~V}$ metal matrix composites, with unidirectional silicon-carbide fiber (Textron SCS-6, with $32 \%$ in fiber volume fraction) in a Ti-6Al-4V matrix under uniaxial transverse tensile normal loading. The experimental data recorded at $23^{\circ} \mathrm{C}$ is used for comparison here. Residual stresses occur by subjecting the composites to cooling from the processing temperature before application of mechanical loads. For simplicity, the silicon-carbide fibers are assumed to be isotropic and homogeneous elastic properties. The elastic moduli of the matrix and fibers as reported by Nimmer et al. (1991) are: $E_{0}=113.7 \times 10^{3} \mathrm{MPa}$, and $v_{0}=0.3$ for the Ti-6Al-4V metal matrix at $21^{\circ} \mathrm{C}$; and $E_{1}=414 \times 10^{3} \mathrm{MPa}$, and $v_{1}=0.3$ for the $\mathrm{SiC}$ fiber. Moreover, 


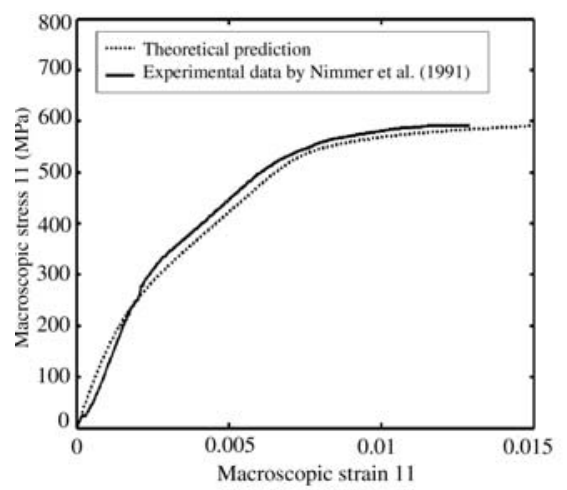

(a)

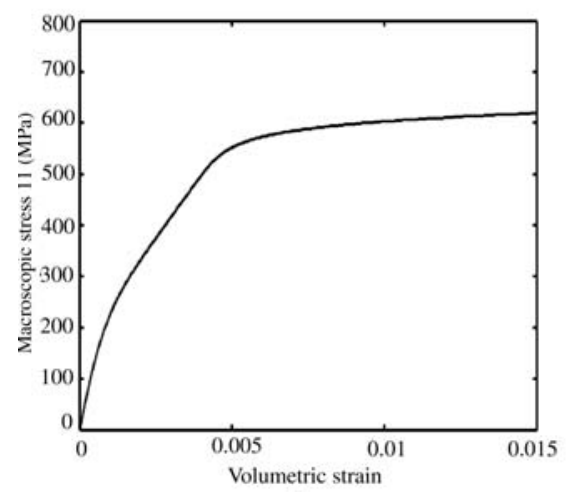

(c)

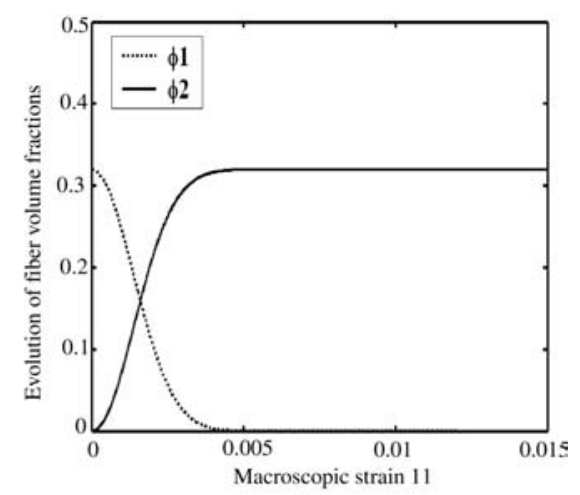

(b)

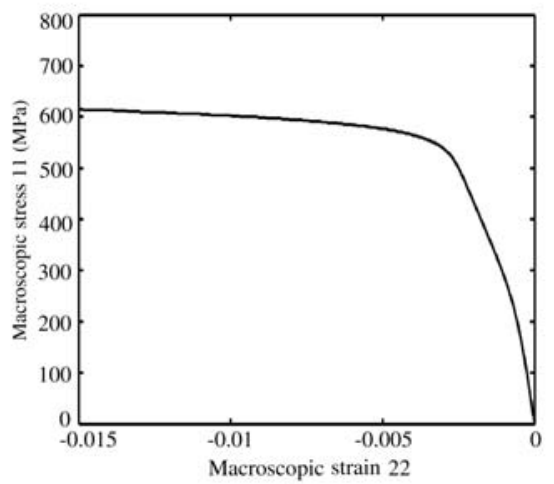

(d)

Figure 3. (a) Comparison of predicted and measured uniaxial transverse stress-strain behavior of SiC/Ti-6Al-4V composites with initial fiber volume fraction $32 \%$, room temperature $=23^{\circ} \mathrm{C}$; (b) corresponding progressive damage of fibers; (c) $\bar{\sigma}_{11}$ vs $\bar{\varepsilon}_{\mathrm{v}}$ (volumetric strain); and (d) $\bar{\sigma}_{11}$ vs $\bar{\varepsilon}_{22}$.

we employ the following plastic parameters for the experimental data at $23^{\circ} \mathrm{C}: \bar{\sigma}_{y}=600 \mathrm{MPa}, h=900 \mathrm{MPa}$, and $q=0.1$. To estimate the Weibull parameters $S_{0}$ and $M$, we follow Ju and Lee $(2000,2001)$ and adopt $S_{0}=210 \mathrm{MPa}$, and $M=2$. The residual stresses due to cooling process are in part considered in our estimation for $S_{0}$ here.

The comparison between the present model predictions and experimental data on the overall uniaxial elastoplastic damage behavior of FRDMCs is shown in Figure 3(a). The solid line corresponds to the experimental data and the dash line corresponds to the current predictions. As depicted in Figure 3(a), the experimental data, at a load substantially less than the measured yield strength, shows a significant reduction in transverse modulus 
taking place. That is, the creation of a characteristic first 'knee' in the transverse tensile stress-strain curve is observed. Physical evidence that the experimentally observed first 'knee' is actually associated with such an interfacial separation event is provided by the edge replica experiments which identify 'gaps' between the fibers and the matrix above the first 'knee' in a transverse loading test (Nimmer et al., 1991). In order to separate the interfaces between the fibers and the matrix, thus creating the first 'knee,' the compressive residual stresses must be overcome by the tensile mechanical tensile loading. At still higher stress level, the matrix plasticity dominates and a maximum stress is reached. Therefore, the second 'knee' is formed. This feature of trilinear-like stress-strain curve can be well captured under the present framework. It also proves that interfacial strength between the fiber and the matrix plays an important role in influencing the overall behavior of FRDMCs. Moreover, Figure 3(b) shows that interfacial damage occurs in the initial steps of the applied loading. The damage gradually increases as the strain increases up to $\bar{\varepsilon}_{11}=0.00454$. Following the next few incremental stress steps, the debonded fiber volume fraction $\phi_{2}$ reaches almost a constant value around $32 \%$; i.e., the composite material now becomes almost a porous ductile medium (with nearly all fibers debonded). In addition, according to the simulation results, at $\bar{\varepsilon}_{11}=0.00392$ and $\bar{\sigma}_{11}=367 \mathrm{MPa}$, the initial yielding of the overall composites occurs. This also explains why the stress-strain curve in Figure 3(a) after $\bar{\varepsilon}_{11}=0.00392$ demonstrates the overall plastic hardening effect. In Figure 3(c) and (d), $\bar{\sigma}_{11}$ versus $\bar{\varepsilon}_{v}$ (the volumetric strain) and $\bar{\sigma}_{11}$ versus $\bar{\varepsilon}_{22}$ corresponding to Figure 3(a) are displayed, respectively. The volumetric strain increases as loading increases after the composite yields. Clearly, the effective yield function in the current framework is pressure-dependent and not of the von Mises type. In addition, due to the Poisson's effect, the $\bar{\varepsilon}_{22}$ strain under $\bar{\sigma}_{11}$ is negative.

The simulations of FRDMCs under biaxial tensile loading with varying stress ratios R are exhibited in Figure 4(a) and (b). In Figure 4(a), the overall $\bar{\sigma}_{11}$ versus $\bar{\varepsilon}_{11}$ is rendered. As the stress ratio $R$ increases, the overall response demonstrates higher stiffness in the elastic range and higher yielding strength of the FRDMCs. Figure 4(b) exhibits the overall relation of transverse $\bar{\sigma}_{22}$ versus $\bar{\varepsilon}_{22}$ at increasing stress ratio $R$. In addition, the 'bend-over' effect becomes more significant as the stress ratio $R$ increases.

\section{CONCLUSIONS}

A micromechanical elastoplastic-damage formulation is developed to predict the overall elastoplastic behavior and interfacial damage evolution of fiber-reinforced ductile matrix composites. To estimate the overall 


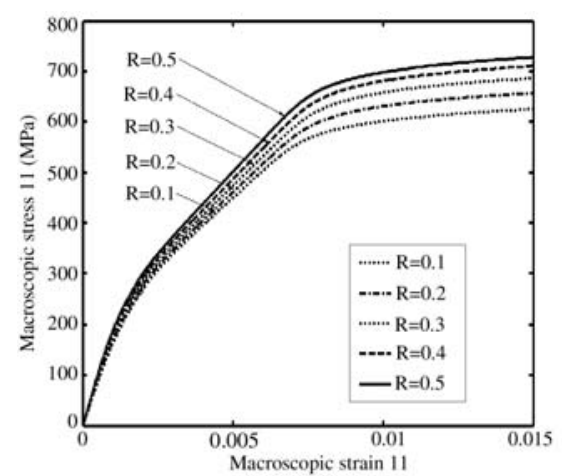

(a)

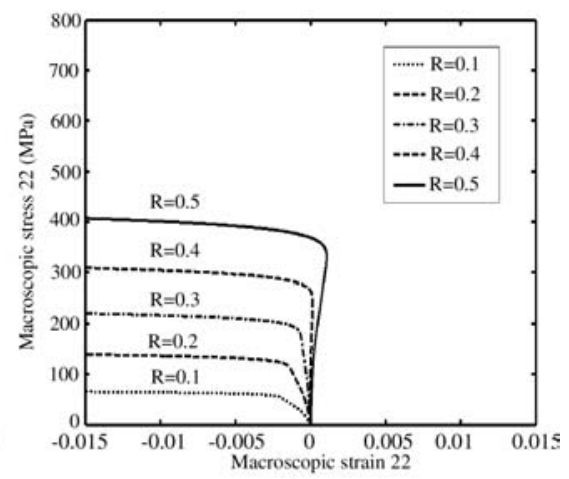

(b)

Figure 4. (a) Overall transverse stress-strain relation $\bar{\sigma}_{11}$ vs $\bar{\varepsilon}_{11}$ of the SiC/Ti-6Al-4V composites with initial fiber volume fraction $32 \%$, room temperature $=23^{\circ} \mathrm{C}$ for various $R$ under biaxial tensile loading and (b) corresponding overall transverse stress-strain relation $\bar{\sigma}_{22}$ vs $\bar{\varepsilon}_{22}$.

elastoplastic damage behavior, an effective yield criterion is micromechanically derived based on the ensemble-area averaging procedure and the first-order effects of eigenstrains due to cylindrical inclusions. The effects of random dispersion of elastic inclusions are considered through the ensemble averaging process. The proposed overall yield criterion, in conjunction with the overall associative plastic flow rule and the hardening law, provide the analytical foundation for the estimation of effective elastoplastic-damage responses. An evolutionary interfacial debonding model is subsequently employed in accordance with the Weibull's probability function to characterize the varying probability of complete fiber debonding. The present uniaxial predictions under the plane strain are also compared with the experimental data reported by Nimmer et al. (1991). The model predictions are generally in good agreement with the experimental data and the salient characteristics of ductile composites.

In a forthcoming study, the effects of partially debonded fibers on the overall behavior of FRDMCs are intended to be further explored in detail. As a result of the partial interfacial debonding, the overall material responses become orthotropic under the uniaxial and biaxial tension loading.

\section{ACKNOWLEDGMENTS}

This work was in part sponsored by the Faculty Research Grant of the Academic Senate of UCLA (Fund Number 4-592565-19914) and by the Bellagio Engineering. 


\section{REFERENCES}

DeBotton, G. and Castañeda, Ponte P. (1993). Elastoplastic Constitutive Relations for Fiberreinforced Solids, Int. J. Solids \& Struct., 30: 1865-1890.

Doghri, I. and Friebel, C. (2005). Effective Elasto-plastic Properties of Inclusion-reinforced Composites. Study of Shape, Orientation and Cyclic Response, Mechanics of Materials, 37: 45-68.

Eshelby, J.D. (1957). The Determination of the Elastic Field of an Ellipsoidal Inclusion, and Related Problems, In: Proc. R. Soc. Lond., A241, pp. 376-396.

Hashin, Z. and Shtrikman, S. (1962). A Variational Approach to the Theory of the Elastic Behavior of Multiphase Materials, J. Mech. Phys. Solids, 11: 127-140.

Hashin, Z. and Rosen, B.W. (1964). The Elastic Moduli of Fiber-reinforced Materials, J. Appl. Mech., 31: 223-232.

Hashin, Z. (1972). Theory of Fiber Reinforced Materials: NASA CR-1974.

Hill, R. (1964a). Theory of Mechanical Properties of Fiber-Strengthened Materials: I. Elastic Behavior, J. Mech. Phys. Solids, 12: 199-213.

Hill, R. (1964b). Theory of Mechanical Properties of Fiber-strengthened Materials: II. Inelastic Behavior, J. Mech. Phys. Solids, 12: 214-218.

Ju, J.W. (1991a). On Two-dimensional Self-consistent Micromechanical Damage Models for Brittle Solids, Int. J. Solids \& Struct., 27(2): 227-258.

Ju, J.W. (1991b). A Micromechanical Damage Model for Uniaxially Reinforced Composites Weakened by Interfacial Arc Microcracks, J. Appl. Mech., 58(4): 923-930.

Ju, J.W. (1996). On Micromechanical Evolutionary Damage Models for Polycrystalline Ceramics, Int. J. Damage Mechanics, 5(2): 113-137.

Ju, J.W. and Chen, T.M. (1994a). Micromechanics and Effective Moduli of Elastic Composites Containing Randomly Dispersed Ellipsoidal Inhomogeneities, Acta Mechanica, 103: $103-121$.

Ju, J.W. and Chen, T.M. (1994b). Effective Elastic Moduli of Two-phase Composites Containing Randomly Dispersed Spherical Inhomogeneities, Acta Mechanica, 103: $123-144$.

Ju, J.W. and Chen, T.M. (1994c). Micromechanics and Effective Elastoplastic Behavior of Two-phase Metal Matrix Composites, J. Eng. Mater. \& Tech., 116: 310-318.

Ju, J.W. and Chen, T.M. (1994d). Effective Elastic Moduli of Two-dimensional Brittle Solids with Interacting Microcracks. Part I: Basic Formulations, J. Appl. Mech., 61: 349-357.

Ju, J.W. and Chen, T.M. (1994e). Effective Elastic Moduli of Two-dimensional Brittle Solids with Interacting Microcracks. Part II: Evolutionary Damage Models, J. Appl. Mech., 61: $358-366$.

Ju, J.W. and Lee, X. (1991). Micromechanical Damage Models for Brittle Solids. Part I: Tensile Loadings, J. Eng. Mech., 117(7): 1495-1515.

Ju, J.W. and Lee, H.K. (2000). A Micromechanical Damage Model for Effective Elastoplastic Behavior of Ductile Matrix Composites Considering Evolutionary Complete Particle Debonding, Computational Methods Appl. Mech. Engrg., 183: 201-222.

Ju, J.W. and Lee, H.K. (2001). A Micromechanical Damage Model for Effective Elastoplastic Behavior of Partially Debonded Ductile Matrix Composites, Int. J. Solids \& Struct., 38(36-37): 6307-6332.

Ju, J.W. and Sun, L.Z. (1999). A Novel Formulation for the Exterior-point Eshelby's Tensor of an Ellipsoidal Inclusion, J. Appl. Mech., 66: 570-574.

Ju, J.W. and Sun, L.Z. (2001). Effective Elastoplastic Behavior of Metal Matrix Composites Containing Randomly Located Aligned Spheroidal Inhomogeneities. Part I: Micromechanics-based Formulation, Int. J. Solids \& Struct., 38: 183-201. 
Ju, J.W. and Tseng, K.H. (1992). A Three-dimensional Statistical Micromechanical Theory for Brittle Solids with Interacting Microcracks, Int. J. Damage Mechanics, 1(1): 102-131.

Ju, J.W. and Tseng, K.H. (1995). An Improved Two-dimensional Micromechanical Theory for Brittle Solids with Many Randomly Located Interacting Microcracks, Int. J. Damage Mechanics, 4(1): 23-57.

Ju, J.W. and Tseng, K.H. (1996). Effective Elastoplastic Behavior of Two-phase Ductile Matrix Composites: a Micromechanical Framework, Inter. J. Solids \& Struct., 33: 4267-4291.

Ju, J.W. and Tseng, K.H. (1997). Effective Elastoplastic Algorithms for Two-phase Ductile Matrix Composites, J. Eng. Mech., 123(3): 260-266.

Ju, J.W. and Zhang, X.D. (1998). Micromechanics and Effective Transverse Elastic Moduli of Composites with Randomly Located Aligned Circular Fibers, Int. J. Solids \& Struct., 35(9-10): 941-960.

Ju, J.W. and Zhang, X.D. (2001). Effective Elastoplastic Behavior of Ductile Matrix Composites Containing Randomly Located Aligned Circular Fibers, Int. J. Solids \& Struct., 38: 4045-4069.

Lee, J. and Mal, A. (1998). Characterization of Matrix Damage in Metal Matrix Composites under Transverse Loads, Computational Mech., 21: 339-346.

Lee, X. and Ju, J.W. (1991). Micromechanical Damage Models for Brittle Solids. Part II: Compressive Loadings, J. Eng. Mech., 117(7), 1516-1537.

Lissenden, C.J. (1996). An Approximate Representation of Fiber-matrix Debonding in Nonperiodic Metal Matrix Composites, In: Damage and Interfacial Debonding in Composites, Elsevier, Amsterdam, pp. 189-212.

Liu, H.T., Sun, L.Z. and Ju, J.W. (2004). An Interfacial Debonding Model for Particlereinforced Composites, Int. J. of Damage Mechanics, 13(2): 163-185.

Mura, T. (1987). Micromechanics of Defects in Solids, 2nd edn, Dordrecht, Martinus Nijhoff.

Nimmer, R.P., Bankert, R.J., Russell, E.S., Smith, G.A. and Wright, P.K. (1991). Micromechanical Modeling of Fiber/matrix Interface Effects in Transversely Loaded SiC/Ti-6-4 Metal Matrix Composites, J. of Comp. Tech. \& Research, 13: 3-13.

Sun, L.Z. and Ju, J.W. (2001). Effective Elastoplastic Behavior of Metal Matrix Composites Containing Randomly Located Aligned Spheroidal Inhomogeneities. Part II: Applications, Int. J. Solids \& Struct., 38: 203-225.

Sun, L.Z. and Ju, J.W. (2004). Elastoplastic Modeling of Metal Matrix Composites Containing Randomly Located and Oriented Spheroidal Particles, J. Appl. Mech., 71: 774-785.

Sun, L.Z., Ju, J.W. and Liu, H.T. (2003a). Elastoplastic Modeling of Metal Matrix Composites with Evolutionary Particle Debonding, Mechanics of Materials, 35: 559-569.

Sun, L.Z., Liu, H.T. and Ju, J.W. (2003b). Effect of Particle Cracking on Elastoplastic Behavior of Metal Matrix Composites, Int. J. for Numer. Meth. in Eng., 56: 2183-2198.

Tohgo, K. and Weng, G.J. (1994). A Progress Damage Mechanics in Particlereinforced Metal-matrix Composites under High Triaxial Tension, J. Eng. Mat. \& Tech., 116: 414-420.

Voyiadjis, G.Z. and Park, T. (1996). Stress and Strain Concentration Tensors for Damaged Fibrous Composites, J. Mech. Behavior Mat., 7(2): 119-138.

Weibull, W. (1951). A Statistical Distribution Function of Wide Applicability, J. Appl. Mech., 18: $293-297$.

Willis, J.R. (1981). Variational and Related Methods for the Overall Properties of Composites, Adv. Appl. Mech., 21: 1-78.

Zhao, Y.H. and Weng, G.J. (1990). Theory of Plasticity for a Class of Inclusion and Fiberreinforced Composites. In Micromechanics and Inhomogeneity, The Toshio Mura 65th Anniversary Volume, Springer-Verlag, New York, pp. 599-622. 
Zhao, Y.H. and Weng, G.J. (1995). A Theory of Inclusion Debonding and its Influence on the Stress-strain Relations of a Ductile Matrix, Int. J. Damage Mech., 4: 196-211.

Zhao, Y.H. and Weng, G.J. (1996). Plasticity of a Two-phase Composite with Partially Debonded Inclusions, Int. J. Plasticity, 12: 781-804.

Zhao, Y.H. and Weng, G.J. (1997). Transversely Isotropic Moduli of Two Partially Debonded Composites, Int. J. Solids \& Struct., 34: 493-507. 